\title{
A New Bivariate Gamma Distribution
}

\author{
Arjun K. Gupta ${ }^{1}$, Dattatraya G. Kabe ${ }^{2}$ \\ ${ }^{1}$ Bowling Green State University, Bowling Green, USA \\ ${ }^{2} 5971$ Greensboro Drive, Mississauga, Canada \\ E-mail: gupta@bgsu.edu
}

Received March 27, 2011; revised May 18, 2011; accepted May 21, 2011

\begin{abstract}
Following Nadarajah [1], we introduce a new bivariate correlated type Gamma distribution, whose joint density is expressed in two parts. Expressions for single and joint moments of the variates are derived. Bivariate Correlated Wishart density follows on similar lines.
\end{abstract}

Keywords: Gamma Distribuion, Correlated Gamma Variates, Moments, Gauss’ Hypergeometric Series

\section{Introduction}

We have joint density of three independent gamma random variables

$$
h(u, v, w)=K u^{a-1} v^{b-1} w^{c-1} \exp \{-(u+v+w)\}
$$

where $K$, as a generic letter, denotes the normalizing constant of a denstiy function in this paper. Setting $u=x-w, u=y-w$ the joint denstiy at $x, y, w$ is

$$
\begin{aligned}
& h(u, v, w) \\
& =K(x-w)^{a-1}(y-w)^{b-1} w^{c-1} \exp \{-(x+y)+w\} .
\end{aligned}
$$

As in Nadarajah [1], if $x<y$, then we set $w=x t$, and if $y<x$, then we set $w=y t$, and integrate out $t$. This is done by using Gradshteyn and Ryzhik ([2], 3.385) result , and we find that (See Equation (3)) where,

$$
\begin{aligned}
\varphi(\alpha, \beta, r, x, y) & =\sum_{j=0}^{\infty} \sum_{k=0}^{\infty} \frac{(\alpha)_{j+k}(\beta)_{k} x^{j} y^{k}}{r_{j+k} j ! k !} \\
& =2 F_{1}(\alpha ; \beta ; r ; x+y)
\end{aligned}
$$

However, Nadarajah [1] does not express the density (3) in terms of $2 F_{1}$.

The series $\varphi(\alpha, \beta, r, x, y)$ is one of the several of Lauricella's series, and Mathai ([3], Theroem 5.59) gives an integral representaion of $\phi$. We use this integral representation to show that $\phi$ can be expressed as $2^{F_{1}}$.

To calculate single or joint moments of $x$ and $y$, we set

$$
\begin{aligned}
& x=\theta_{1} \theta_{2}, y=\theta_{2} ; J\left(x, y: \theta_{1}, \theta_{2}\right)=\theta_{2}, \text { if } x<y \\
& y=\theta_{1} \theta_{2}, x=\theta_{2} ; J\left(x, y: \theta_{1}, \theta_{2}\right)=\theta_{2}, \text { if } x<y .
\end{aligned}
$$

Similarly, if $x<y$, then we set $y=x+z$, $0<x<z$ and integrate out $z$ to obtain the marginal denstiy of $x$. However, if $y<x$, then we must integrate out $y$ from 0 to $x$, by using certain known integral results like

$$
\int_{0}^{x} \exp \{-\alpha y\} y^{p} \mathrm{~d} y=-\frac{p !}{\alpha^{p+1}}\left[\sum_{k=0}^{p} \exp \{-\alpha y\} \frac{(\alpha y)^{k}}{k !}\right]_{0}^{x}
$$

to obtain the marginal density of $x$. However, all such results are already available in advanced calculus books or in the books of collected results on integrals and series.

\section{The Moments}

Mathai [4] shows that the Lauricella's series can be written as

$$
h(x, y)= \begin{cases}K x^{a+c-1} y^{b-1} \exp \{-(x+y)\} \varphi\left(c, 1-b, a+c, \frac{x}{y}, y\right) & \text { if } x<y \\ K x^{a-1} y^{b+c-1} \exp \{-(x+y)\} \varphi\left(c, 1-a, b+c, \frac{y}{x}, x\right) & \text { if } y<x .\end{cases}
$$




$$
\begin{aligned}
F_{D}\left(a, b_{1}, \cdots, b_{n} ; c ; x_{1}, \cdots, x_{n}\right) & =K \int_{0}^{1} u^{a-1}(1-u)^{c-a-1}\left(1-u x_{1}\right)^{-b_{1}} \cdots\left(1-u x_{n}\right)^{-b_{n}} \mathrm{~d} u_{1} \cdots \mathrm{d} u_{n} \\
& =\sum_{r_{1}=0}^{\infty} \cdots \sum_{r_{n}=0}^{\infty} \frac{\Gamma\left(a+r_{1}+\cdots+r_{n}\right) \Gamma\left(b_{1}+r_{1}\right) \cdots \Gamma\left(b_{n}+r_{n}\right)}{\Gamma\left(c+r_{1}+\cdots+r_{n}\right) r_{1} ! r_{2} ! \cdots r_{n} !} x_{1}^{r_{1}} \cdots x_{n}^{r_{n}} .
\end{aligned}
$$

He $([3,4])$ also records several integral representation of the series $F_{D}$. The context one is Mathai ([3], Theorem 5.59, p. 345)

$$
\begin{aligned}
& F_{D}\left(a, b_{1}, \cdots b_{n} ; c ; X_{1}, \cdots, X_{n}\right) \\
& =K \int \exp \left\{-\operatorname{tr}\left(T_{1}+\cdots+T_{n}\right)\right\}\left|T_{1}\right|^{b_{1}-g} \cdots\left|T_{n}\right|^{b_{n}-g_{1}} \\
& \times F_{1}\left(a ; c ; X_{1} T_{1}+\cdots+X_{n} T_{n}\right) \mathrm{d} T_{1} \cdots \mathrm{d} T_{n}
\end{aligned}
$$

$$
\int_{A+B=D} \exp \{-\operatorname{tr}(A+B)\}|A|^{n-g}|B|^{q-g}{ }_{0} F_{1}(n ; \Delta)_{0} F_{1}(q ; \Omega) \mathrm{d} A \mathrm{~d} B=\operatorname{Kexp}\{-\operatorname{tr} D\}|D|_{0 F_{1}}^{n+q-g}(n+q ;(\Delta+\Omega) D),
$$

We write some what incorrectly , but formally the result (10) as

$$
{ }_{0} F_{1}(n ; \Delta A)_{0} F_{1}(q ; \Omega B)={ }_{0} F_{1}(n+q ;(\Delta+\Omega)(A+B)) .
$$

Now from (9)

$$
\begin{aligned}
{ }_{1} F_{1}\left(a ; c ; X_{1} T_{1}+\cdots+X_{n} T_{n}\right) & =\int \exp \{-\operatorname{tr} Z\}|Z|^{a-g_{0}} F_{1}\left(c ; X_{1} T_{1}+\cdots+X_{n} T_{n}\right) \mathrm{d} Z \mathrm{~d} T_{1} \cdots \mathrm{d} T_{n} \\
& =\int \exp \{-\operatorname{tr} Z\}|Z|^{a-g_{0}} F_{1}\left(c_{1} ; X_{1} T_{1} Z\right) \cdots{ }_{0} F_{1}\left(c_{n} ; X_{n} T_{n} Z\right) \mathrm{d} Z \mathrm{~d} T_{1} \cdots \mathrm{d} T_{n} \\
& =\int \exp \{-\operatorname{tr} Z\}|Z|^{a-g_{0}} F_{1}\left(c ;\left(X_{1}+X_{2}+\cdots+X_{n}\right)\left(T_{1}+T_{2}+\cdots+T_{n}\right) Z\right) \mathrm{d} Z \mathrm{~d} T_{1} \cdots \mathrm{d} T_{n} \\
& =\int \exp \{-\operatorname{tr} Z\}|Z|^{a-g_{0}} F_{1}\left(c_{1} ; X_{1} T_{1} Z\right) \cdots{ }_{0} F_{1}\left(c_{n} ; X_{n} T_{n} Z\right) \mathrm{d} Z \mathrm{~d} T_{1} \cdots \mathrm{d} T_{n} \\
& ={ }_{1} F_{1}\left(a ; c ;\left(X_{1}+X_{2}+\cdots+X_{n}\right)\left(T_{1}+T_{2}+\cdots+T_{n}\right)\right)
\end{aligned}
$$

where $c=c_{1}+\cdots+c_{n}$ and hence from (9) and (12) we have

$$
F_{D}\left(a ; b_{1}, \cdots, b_{n} ; c ; X_{1}, X_{2}, \cdots, X_{n}\right)={ }_{2} F_{1}\left(a ; b_{1}+\cdots+b_{n} ; c ; X_{1}+X_{2}+\cdots+X_{n}\right) .
$$

The marginal density of $x$, if $x<y$, setting $y=x+z$, is

$$
\begin{aligned}
h(x) & =K \int(x-w)^{a-1}(x-w+z)^{b-1} \exp \{-2 x+z+w\} \mathrm{d} z \mathrm{~d} w \\
& =K \sum_{r=0}^{\infty} \frac{\Gamma(b+r)}{r !} \int(x-w)^{a+r-1} z^{b+r-1} w^{c-1} \exp \{-2 x-z+w\} \mathrm{d} z \mathrm{~d} w \\
& =K \sum_{r=0}^{\infty} \frac{\Gamma(b+r) \Gamma(b+r)}{r !} \exp \{-2 x\}_{1} F_{1}(c ; a+c+r ; x)
\end{aligned}
$$

Similarly, using (7) we can find the marginal density of $x$, when $x<y$. Then using (5),(6) and (14) we find that

$$
\begin{aligned}
E\left(x^{m} y^{n} \mid x<y\right)+E\left(x^{m} y^{n} \mid y<x\right) & ={ }_{2} F_{1}\left(1, b+n ; m+n+c+1 ; \frac{1}{2}\right)_{3} F_{1}(c,(1-b) ; m+n+c+b ; a+c ; 1) \\
& +{ }_{2} F_{1}\left(1, a+n ; m+n+c+1 ; \frac{1}{2}\right)_{3} F_{1}(c,(1-a) ; m+n+c+a ; b+c ; 1)
\end{aligned}
$$

The integral in (15) is evaluated by using first (5), (6), and then

$$
\int_{0}^{1} x^{m-1}(1-x)^{n-1}(1+\alpha x)^{-(m+n+p)} \mathrm{d} x=K(1+\alpha)_{2}^{-(m+n+p)} F_{1}\left(n ; 1 ; m+n ; \frac{\alpha}{1+\alpha}\right) .
$$


Note that (15) consists of a double series and not a triple series as in Nadarajah ([1], Section 3). Although he ([1], Section 5) wishes to generalize bivariate correlated gamma densities to $n$ variate similar gamma densities, it is not a simple task. It will involve complicated order statistics theory from different Populations.

\section{References}

[1] S. Nadarajah, “A New Bivariate Chi-Distribution,” SIAM Review, 2006, (unpublished).
[2] I. S. Gradsheleyn and I. M. Ryzhik, "Tables of Integral, Series, and Products,” 5th Edition, Academic Press, New York, 2000.

[3] A. M. Mathai, "Jacobian of Transformations and Functions of Matrix Argument. World Scientific,” London, 1997.

[4] A. M. Mathai, “A Handbook of Generalized Special Functions for Statistical and Physical Sciences,” Claredon Press, Oxford, 1993. 